\title{
The Target Vehicle Movement State Estimation Method with Radar Based on Kalman Filtering Algorithm
}

\author{
Jia Xin \\ State Key Lab of Automobile \\ Simulation and Control \\ Jilin University \\ Changchun, China \\ e-mail: jia.xin@ascl.jlu.edu.cn
}

\author{
Wu Zuolong \\ State Key Lab of Automobile \\ Simulation and Control \\ Jilin University \\ Changchun, China \\ e-mail: wu.zuolong@ascl.jlu.edu.cn
}

\author{
Guan Hsin \\ State Key Lab of Automobile \\ Simulation and Control \\ Jilin University \\ Changchun, China \\ e-mail: guan.hsin@ascl.jlu.edu.cn
}

\begin{abstract}
In this paper, based on Kalman filtering algorithm, a method of target vehicle motion state radar estimation with radar(or lidar) is presented. The state equations is established based on rigid plane dynamics theory, and then with a Kalman filter to do radar data processing, the position, velocity and acceleration of the target vehicle can be estimated at the same time, so that to cover the shortage that acceleration information can not be gained with radar system. Through simulation and field tests it is verified that the detection accuracy of position and velocity of target vehicle is increasing, and the acceleration of target vehicle can be estimated effectively and accurately.
\end{abstract}

Keywords-intelligent vehicle; radar(lidar); target vehicle; acceleration estimation; Kalman filter

\section{INTRODUCTION}

Driving assistant systems for automobile to assist driver to make the correct driving decisions, it is necessary to obtain the information of the vehicle movement state accurately around obstacles, including position, velocity, and acceleration. With radar (or lidar) only the information of the target vehicle (obstacle) can be obtained is the position and velocity in the carrier coordinate system of radar.

With the published literature, domestic and foreign research on radar signal processing almost using low-order (two or three-order) Kalman filtering method of the radar measurement signal processing, are assuming the same speed or acceleration of the target vehicle so that to estimates the position and the speed of target vehicle with Kalman filter.

For examples, Wang Rongben from Jilin University in 2002 [1], Xiang Zhiyu from Zhejiang University in 2005 [2], Yu Chunhe from Zhejiang University in 2006 [3], Masafumi from Doshisha University in 2008 [4], J. Paul from German in 2010 [5], Mai Xinchen from Shanghai Jiao Tong University in 2011 [6], Zhao Wanli from Central South University in 2011 [7], Shen Ling from Nanjing University of Science and Technology in 2012 [8], , Yang Fei from Zhejiang University in 2012 [9], and Navlab Group from Carnegie Mellon University 2012 [10], are all making the hypothesis that the acceleration of target vehicle are constant, and then the relative distance and speed according to radar carrier can be observed. Kalman filtering is used to estimate movement states of target vehicle, including relative distance and speed according to radar carrier, with state equations based on rigid body dynamics principles. Since acceleration of target vehicle is not estimated, the movement state observed is not accurate enough.

With traditional radar information processing results above, it can only assume that the target vehicle will do uniform linear motion in the next cycle of information collection, so that to determine its trajectory of the vehicle relative to radar carrier, and then to determine whether the car will be safe or not while moving. Obviously, the accuracy of this method above in the high-speed host driving vehicle (intelligent vehicle), so it can't be guaranteed to make accurate judgment of the safety of host vehicle, resulting in a potential accident hazard. In the paper a method is presented based on Kalman filtering with radar system to estimate movement state of target vehicle. Combined with the movement state information radar carrier (intelligent vehicle), which can be detected with GPS/INS, the position, velocity and acceleration can be estimated at the same time with Kalman filter, through state equations established on the basis of rigid plane dynamics theory. Through this method, not only to effectively improve the detection accuracy for target vehicle position and velocity, but also to present a reliable estimation for target vehicle acceleration at the same time, thereby to be able to give a more accurate trajectory for intelligent vehicle moving to be used to make control strategy decisions, so that to ensure intelligent vehicle driving safely and efficiently.

\section{PRINCIPLE OF KALMAN FILTERING}

Linear system can usually be described with state equations, observation equations and initial conditions. Here the state equation of linear discrete system is as follows [11]:

$$
\mathrm{X}(\mathrm{k}+1)=\Phi(\mathrm{k}) \mathrm{X}(\mathrm{k})+\mathrm{B}(\mathrm{k}) \mathrm{u}(\mathrm{k})+\mathrm{W}(\mathrm{k})
$$

Further more, it is assumed that the observation model is set as follow:

$$
\mathrm{Z}(\mathrm{k})=\mathrm{H}(\mathrm{k}) \mathrm{X}(\mathrm{k})+\mathrm{V}(\mathrm{k})
$$

Wherein, $\mathrm{X}(\mathrm{k})$ is the state vector of target at time point $\mathrm{k}$, $\Phi(\mathrm{k})$ is the state transition matrix, $\mathrm{B}(\mathrm{k})$ is the control weighting matrix of system input, $\mathbf{u}(\mathrm{k})$ is the input control signal, $\mathbf{Z}(\mathrm{k})$ is the observation value, $\mathrm{H}(\mathrm{k})$ is the measurement matrix, and $W(\mathbf{k})$ and $V(\mathbf{k})$ are white noise 
which are zero-mean and independent to each other, and are satisfying the following conditions:

$$
\left\{\begin{array}{c}
E\left[w_{k} w_{j}^{T}\right]=Q_{k} \delta_{k, j} \\
E\left[v_{k} v_{j}^{T}\right]=R_{k} \delta_{k, j} \quad \text { for } a l l k, j \\
E\left[v_{k} w_{t}^{T}\right]=0
\end{array}\right.
$$

To the filter used in the practical project, $R_{k}$, the covariance of measurement noise, can generally be obtained by observation. So it is the known conditions of the filter. $R_{k}$ can be calculated with some system observation values from offline.

According to the state equation, the prediction of the state at time point $\mathrm{k}$ with time point $\mathrm{k}-1$ is as follow:

$$
x_{k}^{-}=\Phi_{k-1} x_{k-1}+B_{k-1} u_{k-1}
$$

When new observation $\mathrm{Z}(\mathrm{k})$ is coming, it will be changed to:

$$
\begin{gathered}
\hat{x}_{k}=\left(\Phi_{k-1} \hat{x}_{k-1}+B_{k-1} u_{k-1}\right) \\
+K_{k}\left[z_{k}-H_{k}\left(\Phi_{k-1} \hat{x}_{k-1}+B_{k-1} u_{k-1}\right)\right]
\end{gathered}
$$

Where $K_{k}$ is the filter gain, which can be determined with the minimum mean square error principle as follow:

$$
K_{k}=P_{k}^{-} H_{k}^{T}\left(H_{k} P_{k}^{-} H_{k}^{T}+R_{k}\right)^{-1}
$$

Where $P_{k}^{-}$is the prediction error variance matrix:

$$
\begin{aligned}
P_{k}^{-} & =E\left[\left(x_{k}-z_{k}^{-}\right)\left(x_{k}-z_{k}^{-}\right)^{T}\right] \\
& =\Phi_{k-1} P_{k-1} \Phi_{k-1}^{T}+Q_{k-1}
\end{aligned}
$$

Where $P_{k}$ is the forecast error variance matrix for $\hat{x}_{k}$ :

$$
\begin{aligned}
P_{k} & =E\left[\left(x_{k}-\hat{x}_{k}\right)\left(x_{k}-\hat{x}_{k}\right)^{T}\right] \\
& =\left[\left(P_{k}^{-}\right)^{-1}+H_{k}^{T} R_{k}^{-1} H_{k}\right]^{-1}
\end{aligned}
$$

\section{Estimation OF TARget Vehicle}

The relative movement state of the target vehicle at time point $\mathrm{k}$, given by the radar, is that the position is $\left[x_{k}, y_{k}\right]^{T}$ and the velocity is $\left[v_{x k}, v_{y k}\right]^{T}$ in the reference system of the vehicle. The movement state of the host vehicle (radar carrier) can be given with GPS. In the process from time point $\mathrm{k}$ to $\mathrm{k}$ +1 , the movement of the host vehicle along the $\mathrm{x}$-axis and $\mathrm{y}$ axis respectively is $\Delta x_{k}$ and $\Delta y_{k}$, and the value of the heading angle changing is $\theta_{k}$. So the new coordinates in vehicle reference system at time point $k+1$ can be achieved according to the knowledge of the translation and rotation transformation of the coordinates shown in Figure 1 and calculated as follow:

$$
\left\{\begin{array}{c}
x_{k+1}=\left(x_{k}-\Delta x_{k}+v_{x k} T+\frac{T^{2}}{2} a_{x k}\right) \cos \theta_{k} \\
-\left(y_{k}-\Delta y_{k}+v_{y k} T+\frac{T^{2}}{2} a_{y k}\right) \sin \theta_{k} \\
y_{k+1}=\left(x_{k}-\Delta x_{k}+v_{x k} T+\frac{T^{2}}{2} a_{x k}\right) \sin \theta_{k} \\
+\left(y_{k}-\Delta y_{k}+v_{y k} T+\frac{T^{z}}{2} a_{y k}\right) \cos \theta_{k}
\end{array}\right.
$$

The velocity of target vehicle in the new reference system is that:

$$
\left\{\begin{array}{c}
v_{x k+1}=\left(v_{x k}+a_{x k} T\right) \cos \theta_{k} \\
-\left(v_{y k}+a_{y k} T\right) \sin \theta_{k} \\
v_{y k+1}=\left(v_{x k}+a_{x k} T\right) \sin \theta_{k} \\
+\left(v_{y k}+a_{y k} T\right) \cos \theta_{k}
\end{array}\right.
$$

So the acceleration of target vehicle in the new reference system is as follow:

$$
\left\{\begin{array}{l}
a_{x k+1}=a_{x k} \cos \theta_{k}-a_{y k} \sin \theta_{k} \\
a_{y k+1}=a_{x k} \sin \theta_{k}+a_{y k} \cos \theta_{k}
\end{array}\right.
$$

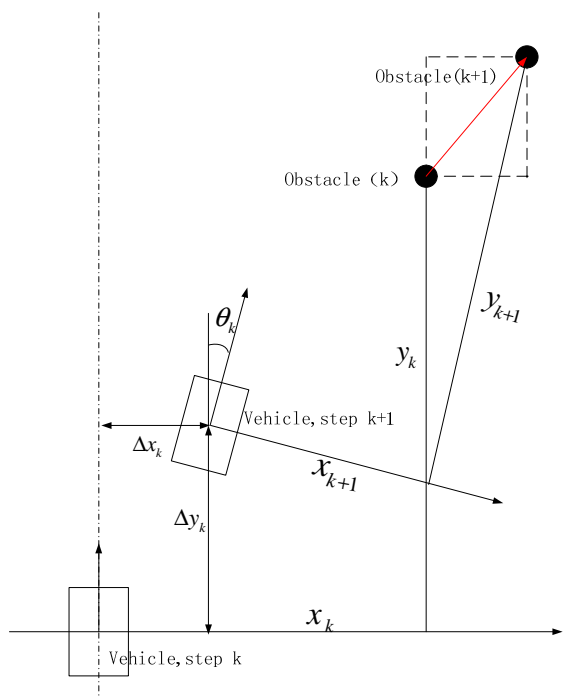

Figure 1. The movement of target vehicle

The Kalman filter is established as follow:

The observation matrix is defined as:

$$
X_{k}=\left[x_{k}, y_{k}, v_{x k}, v_{y k}, a_{x k}, a_{y k}\right]^{T}
$$

Then it can be obtained that:

$\mathrm{X}(\mathrm{k}+1)=\Phi(\mathrm{k}) \mathrm{X}(\mathrm{k})+\mathrm{B}(\mathrm{k}) \mathrm{u}(\mathrm{k})+\mathrm{W}(\mathrm{k})$

Then the system transition matrix is as follow:

$\Phi(\mathrm{k})=\left[\begin{array}{cccccc}\cos \theta_{k} & -\sin \theta_{k} & T \cos \theta_{k} & -T \sin \theta_{k} & \frac{T^{2}}{2} \cos \theta_{k} & -\frac{T^{2}}{2} \sin \theta_{k} \\ \sin \theta_{k} & \cos \theta_{k} & T \sin \theta_{k} & T \cos \theta_{k} & \frac{T^{2}}{2} \sin \theta_{k} & \frac{T^{2}}{2} \cos \theta_{k} \\ 0 & 0 & \cos \theta_{k} & -\sin \theta_{k} & T \cos \theta_{k} & -T \sin \theta_{k} \\ 0 & 0 & \sin \theta_{k} & \cos \theta_{k} & T \sin \theta_{k} & T \cos \theta_{k} \\ 0 & 0 & 0 & 0 & \cos \theta_{k} & -\sin \theta_{k} \\ 0 & 0 & 0 & 0 & \sin \theta_{k} & \cos \theta_{k}\end{array}\right]$

The input control weighting matrix is as follow:

$$
\mathrm{B}(\mathrm{k})=\left[\begin{array}{cc}
-\cos \theta_{k} & \sin \theta_{k} \\
-\sin \theta_{k} & -\cos \theta_{k} \\
0 & 0 \\
0 & 0 \\
0 & 0 \\
0 & 0
\end{array}\right]
$$

The system input matrix is that $u_{k}=\left[\Delta x_{k}, \Delta y_{k}\right]^{T}$

The observation vector is that $Z_{k}=\left[x_{k}, y_{k}, v_{x k}, v_{y k}\right]^{T}$

The observation noise is named as $V_{k}$ 
The measurement matrix is as follow:

$$
H(k)=\left[\begin{array}{llllll}
1 & 0 & 0 & 0 & 0 & 0 \\
0 & 1 & 0 & 0 & 0 & 0 \\
0 & 0 & 1 & 0 & 0 & 0 \\
0 & 0 & 0 & 1 & 0 & 0
\end{array}\right]
$$

Then the estimation of position, velocity and acceleration of target vehicle can be achieved.

\section{TESting AND ANALYZING}

\section{A. Simulation Testing}

The simulation conditions are set as follow:

a) The target vehicle: the initial position at $100 \mathrm{~m}$ in front of the host vehicle, the initial velocity is $28 \mathrm{~m} / \mathrm{s}$, and the acceleration is $3.2 \mathrm{~m} / \mathrm{ss}$; it will move as a straight line with the movement state as above where the acceleration is constant for $5 \mathrm{~s}$, and then will move with the constant speed for $5 \mathrm{~s}$.

b) The host vehicle: The initial velocity is $30 \mathrm{~m} / \mathrm{s}$, and the acceleration is $1 \mathrm{~m} / \mathrm{ss}$; it will move as a straight line the same as target vehicle.

c) The position, velocity and acceleration of target vehicle which are relative to the host vehicle will be estimated at real time with Kalman filter method above.

In order to simulate the effect of real sensor detection, the interference noise is added into the data of relative distance and relative speed between the two vehicles, as the detection data of radar, shown as Figure 2 and Figure 3. (The blue line is ideal value, and the red one is real value with noise, the same below.)

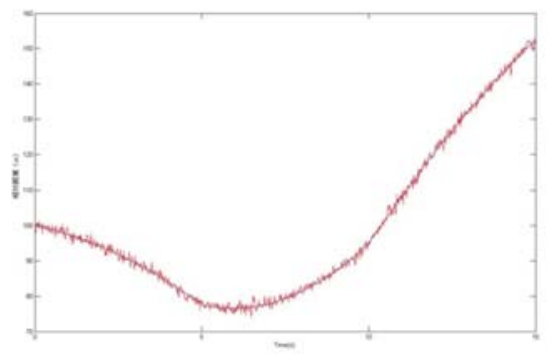

Figure 2. Relative Distance

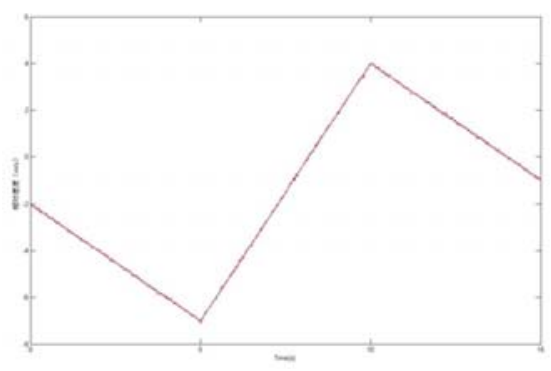

Figure 3. Relative Speed

The relative velocity and acceleration estimations between host vehicle and target vehicle which are obtained with Kalman filter, and the errors between them and ideal value which is set in simulation, are shown in Figure 4 and
Figure 5 below. It can be seen that the estimation results are approximately consistent with the ideal data, but there is some certain fluctuation and delay, which may be seen as caused with interference noise. And there is some small error between them, that the velocity error is in $-2 \mathrm{~m} / \mathrm{s}$ to $+2 \mathrm{~m} / \mathrm{s}$, and acceleration error is between to $-3 \mathrm{~m} / \mathrm{ss}+$ and $3 \mathrm{~m} / \mathrm{ss}$.
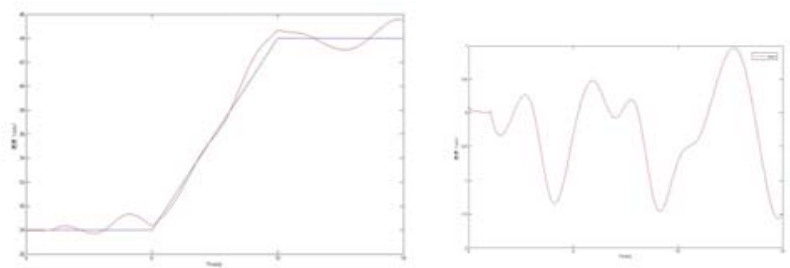

Figure 4. Velocity comparison of estimation result and ideal date and its error


Figure 5. Acceleration comparison of estimation result and ideal date and its error

According with the comparison results, it is proved that the Kalman filtering method to estimate movement state of target vehicle is reasonable and effective.

\section{B. Performance Testing of Algorithm in This Paper}

Here the field testing is established as comparison with detection data from electro-optical speedometer setting on the target vehicle and Kalman filter estimation of radar data setting on the host vehicle. Since the electro-optical speedometer is a kind of high-precision measuring instruments to velocity and acceleration, its detection data is looked as the ideal value in the experiment. Compared with the estimation results from Kalman filter, the feasibility of the method presented in the paper will be proved. The conditions of the field testing is as that: the target vehicle is moving in the front, and the host vehicle is following; both of them are moving along with its own lane independently, but the distance between them must within the detecting region of IBEO LUX Lidar from German used in the paper.

The experimental data is shown that the movement state of target vehicle with Kalman filter from radar is certain consistent with the detection results with electro-optical speedometer, so that it is gained that the estimation results with Kalman filter can show the value and change process of target vehicle velocity and acceleration, so the effectiveness of the method in the paper can be proved. 


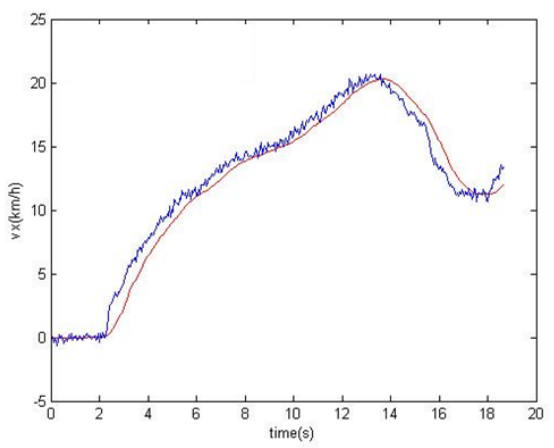

Figure 6. Comparison of longitude velocity

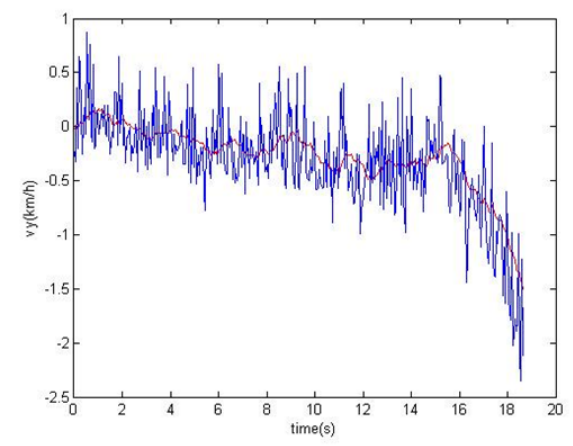

Figure 7. Coparison of latitude velocity

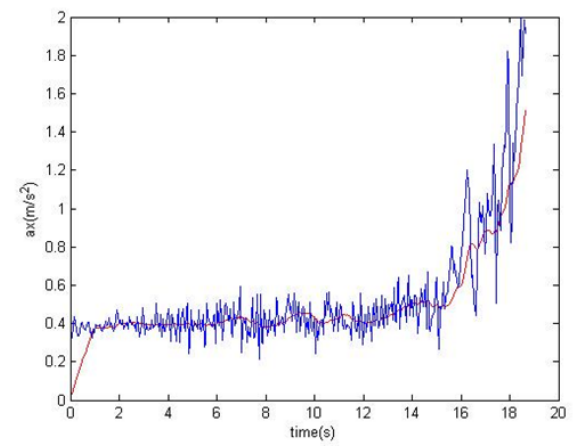

Figure 8. Comparison of longitude acceleration

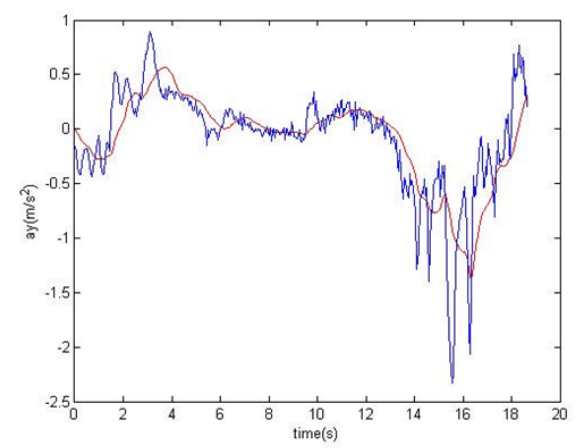

Figure 9. Comparison of latitude acceleration

\section{CONCLUSION}

With the method in the paper, the position, velocity and acceleration can be estimated at the same time through Kalman filter based on the relative position and velocity information provided with radar system, , so that the more accurate movement state of target vehicle can be achieved effectively. And the simulation and field tests are established. In the simulation test, the effectiveness of method is proved with angle-step conditions testing of vehicle steering. In the field test, the feasibility of method is proved with the comparison of estimation results of method and data record of photoelectric speedometer and gyro meter on vehicle. The results of tests can prove that with method in the paper, the accuracy of position and velocity detection of target vehicle is increased, and the acceleration of target vehicle is estimated reliably at the same time. So the more accurate moving tracking of target vehicle can be estimated, which will be used in intelligent vehicle control to drive safely and efficiently.

\section{ACKNOWLEDGMENT}

The study in the paper is supported with Program for Changjiang Scholars and Innovative Research Team in University (No. IRT0626).

\section{REFERENCES}

[1] Wang Rongben, Li Bin, Chu Jiangwei. A Review of Safety Guarantee Technology of Worldwide Intelligent Vehicle, Journal of Highway and Transportation Research and Development, vol. 19, No. 2, 50-54.

[2] Xiang Zhiyu. Obstacle detecting system for off-road autonomous navigation, Journal of Southeast University (Natural Science Edition), vol. 35, Sup II, 71-74.

[3] Yu Chunhe, Chen Guobin, Liu Jilin. Obstacle detection and map reconstruction using multi-layer laser radar, Journal of Zhejiang University (Engineering Science), vol. 40, No. 6, 1066-1069.

[4] Masafumi. Moving object tracking with multi-laser range sensors for mobile robot navigation, Proceedings of IEEE International Conference on Robotics and biomimetics,2007:399-403.

[5] J.TH. Paul. Vehicle system dynamics estimation using Kalman Filter, International Journal of Vehicle Mechanics and Mobility,2010,09:171-183.

[6] Mai Xinchen. Multi-sensor Fusion Based Vehicle Detection and Tracking, Master Dissertation, Shanghai Jiao Tong University, 2011.

[7] Zhao Wanli. Technology Research of the Multi-objective Detection and Tracking for Intelligent Vehicle Based on Radars, Master Dissertation, Central South University, 2011.

[8] Shen Ling. Study on radar data processing with software, Master Dissertation, Nanjing University of Science and Technology, 2012.

[9] Yang Fei. Moving objects detection and tracking with 3D lidar, Master Dissertation, Zhejiang University, 2012.

[10] M.Christoph, E. Luise, et al. Moving object detection with laser scanner,Journal of Field Robotics,2012(10):1-27.

[11] Kalman R E. A New Approach to Linear Filtering and Prediction Theory, Trans.ASME, Journal of Basie Eng,1960. 